\title{
El tránsito de Venus de 2012 visible desde Honduras: Una oportunidad de entender nuestro lugar en el Universo
}

María Cristina Pineda de Carías

\section{Resumen}

Los tránsitos de Venus son eventos de gran interés para la ciencia. Ocurren cuando el planeta Venus se alinea e interpone entre la Tierra y el Sol, produciendo para nosotros el efecto visual de que un muy pequeño objeto negro atraviesa la superficie solar. Su entendimiento, desde los primeros tiempos estuvo ligado al conocimiento de la estructura y dimensiones del Sistema Solar. La frecuencia con que ocurren puede estar separada hasta por más de 100 años, motivo por el cual su observación los convierte en raros eventos que cada vez que vuelven a ser visibles, encuentra a los científicos de diferentes siglos con nuevos instrumentos y más sofisticadas tecnologías. En este artículo, hacemos un repaso histórico desde cuando se predijo la primera posible ocurrencia de un tránsito de Venus, documentando además otras veces que se pudo observar por algunos astrónomos desde diferentes latitudes del planeta, describiendo las circunstancias, limitaciones, equipos y lugares que les permitió registrar y documentar tales observaciones. En la parte final se explica el gran reto que para Honduras ha representado, particularmente para la Facultad de Ciencias Espaciales por medio de su Departamento de Astronomía y Astrofísica, llegar a informar a la población del tipo de evento astronómico observado en junio de 2012, conseguir que el mayor número de personas se interesara por observarlo de manera segura, e igualmente importante, registrar imágenes de cómo los astrónomos nacionales lo observamos, dejando un legado de referencia para los astrónomos que en 2125 nuevamente lo podrán observar.

Palabras clave: Sistema Solar, tránsito de Venus, historia observaciones astronómicas, Honduras. 


\section{Abstract}

Transits of Venus are events of great interest to science. They occur when the planet Venus is aligned between Earth and the Sun, producing for us visual effect that a very small black object passes through the solar surface. His understanding, from the earliest times was linked to the knowledge of the structure and dimensions of the solar system. The frequency with which they occur can be separated up to more than 100 years, reason why becomes increasingly rare events whenever they return to be visible, find scientists of different centuries with new instruments and more sophisticated technologies. In this paper, we make a historical review from when the earliest occurrence of a transit of Venus was predicted further documenting sometimes could be observed by astronomers from different latitudes of the planet, describing the circumstances, limitations, equipment and places they allowed to register and document these observations. The final part describes the great challenge that Honduras has represented, particularly for the Faculty of Space Sciences through its Department of Astronomy and Astrophysics, get to inform people of the type of astronomical event observed in June 2012, getting explained the more people become interested in observing safely, and equally important, an image of how national astronomers observed, leaving a legacy of reference for astronomers in 2125 it will see again.

Keywords: Solar System, transit of Venus, history astronomical observations, Honduras.

María Cristina Pineda de Carías (mcpinedacarias@gmail.com) Universidad Nacional Autónoma de Honduras. Facultad de Ciencias Espaciales. 


\section{Introducción}

Los tránsitos de Venus son eventos raros de gran interés para la ciencia, la investigación y para la educación científica en general. Ocurren cuando el planeta Venus se interpone y alinea entre la Tierra y el Sol, produciendo para nosotros en la Tierra el efecto visual de que un objeto circular negro muy pequeño atraviesa la superficie solar. Los tránsitos de Venus se pueden observar cada cien años en pares, separados por unos ocho años. Son visibles cada vez solo desde determinados lugares de la Tierra. Desde el descubrimiento del telescopio solamente seis eventos han ocurrido, en 1631, 1639, 1761, 1769, 1874 y 1882. En la pareja de tránsitos de Venus de este siglo, el primero ocurrió el 8 de junio de 2004 pero no fue visible desde Honduras. El segundo el tránsito ocurrió el 5 de junio de 2012, siendo visible desde todo el territorio hondureño.

En el tránsito de junio de 2012, a Venus le tomó unas 6 horas con 30 minutos atravesar el lado norte del Sol. Se llama I Contacto al momento cuando el planeta toca aparentemente la parte exterior del disco Solar. El II Contacto ocurre cuando visualmente el planeta está completamente inmerso en la superficie solar tocando el borde del limbo solar. El III Contacto es cuando el planeta, a punto de dejar la superficie solar, toca de nuevo el limbo solar. EI IV Contacto ocurre cuando el planeta ya ha egresado de la superficie solar pero toca aparentemente el borde exterior del Sol. En Honduras, el tránsito de Venus fue visible por la tarde, el I Contacto ocurrió a las 16h: $10 \mathrm{~m}$ y el II Contacto a las $16 \mathrm{~h}: 28 \mathrm{~m}$. Dado que la puesta del Sol fue a las 18h: $15 \mathrm{~m}$, no fue posible observar la totalidad del evento. El III y IV Contactos ocurrieron en la noche a las $22 \mathrm{~h}: 32 \mathrm{~m}$ y a las $22 \mathrm{~h}: 45 \mathrm{~m}$ respectivamente. Los tránsitos de Venus son raros porque tienen un extraño patrón de frecuencia que sucede cada 121 1/2 años, 8 años, 105 1/2 años, 8 años, período al cabo del cual se repite el patrón. Esto es debido a que las órbitas respecto al Sol de la Tierra y de Venus tienen diferentes inclinaciones.

La siguiente pareja de tránsitos de Venus ocurrirá en los años de 2117 y 2125. El tránsito de Venus del 11 de diciembre de 2117 no será visible desde Honduras. En cambio el tránsito de Venus del 8 de diciembre de 2125 si será visible desde todo el territorio, desde su inicio hasta su terminación. Así pues, será hasta dentro de 113 años que los hondureños volveremos a observar un tránsito de Venus. Para esa fecha, las nuevas generaciones, además de los nuevos conocimientos y tecnologías de que dispongan, contarán también con la documentación que para ellos les deja la Facultad de Ciencias Espaciales de la Universidad Nacional Autónoma de Honduras. Este Volumen de la Revista Ciencias Espaciales recoge y describe las diferentes actividades y observaciones realizadas por profesores y estudiantes de sus departamentos de Astronomía y Astrofísica, Arqueoastronomia 
y Astronomía Cultural, del Observatorio Astronómico Centroamericano de Suyapa, y de todas las dependencias universitarias que hicieron posible llegar hasta casi un millón de personas.

En este artículo, a partir de una revisión bibliográfica especializada, se presenta un repaso histórico de las veces en que los tránsitos de Venus se han podido observar, describiendo a los astrónomos involucrados, las circunstancias, condiciones, equipos, lugares y resultados alcanzados. Se presentan también los retos que para Honduras, particularmente para la Facultad de Ciencias Espaciales ha representado la difusión del evento, la orientación a la población para la observación segura, y la documentación mediante imágenes astronómicas de la ocurrencia del evento visto desde el territorio nacional.

\section{Reseña histórica de los tránsitos de Venus observados}

El entendimiento de los tránsitos de Venus, desde los primeros tiempos ha estado ligado al conocimiento de la estructura y dimensiones del Sistema Solar. Considerados históricamente importantes han permitido, conocer la escala del Sistema Solar, la existencia de la atmósfera de Venus, y recientemente, aplicar la técnica de los tránsitos para descubrir planetas extrasolares. Lo que sigue es una relación de los hechos históricos de las observaciones de los tránsitos de Venus.

\section{Pareja 1631 - 1639}

La historia comienza cuando el astrónomo alemán Johannes Kepler (15711630) predijo por primera vez la ocurrencia de los tránsitos de Mercurio y de Venus. La predicción se basó en el modelo del Sistema Solar en que estos dos planetas se mueven alrededor del Sol en órbitas menores e interiores a la órbita de la Tierra. Sus cálculos de los movimientos planetarios, basados en las abundantes observaciones heredadas de Tycho Brahe, además de calcular las proporciones entre las distancias de los planetas conocidos (Mercurio, Venus, Tierra, Marte, Júpiter y Saturno), le permitieron predecir con precisión el tránsito de Mercurio del 7 de noviembre de 1631 y el tránsito de Venus justo un mes después. Lamentablemente Kepler murió antes de que estos eventos ocurrieran, no teniendo la oportunidad de confirmar sus propias predicciones.

La noticia de los tránsitos de Mercurio y de Venus se difundió en Europa. El científico y sacerdote Pierre Gassendi observó el tránsito de Mercurio, convirtiéndose en el primer ser humano que miró un tránsito planetario con un claro entendimiento de lo que exactamente él estaba viendo. Gassendi también trató de 
observar el tránsito de Venus, pero el tránsito ya había terminado antes de que el Sol se levantara en su lugar en París, porque el tránsito no era visible en Europa. Tristemente se dice entonces que, no hay documentación de que alguien en el mundo haya observado el tránsito de Venus de 1631.

Se atribuye al joven Jeremiah Horrocks, feligrés de la Iglesia de San Miguel, en el pueblo de Hoole, cerca de Liverpool en Inglaterra, ser la primera persona que observó un tránsito de Venus en 1639. Horrocks notó que Johannes Kepler no había incluido en las Tablas Rudolfinas, con datos estelares y planetarios, la predicción del tránsito de Venus. Con varias semanas de anticipación, hizo los cálculos matemáticos del día y hora aproximada en que ocurriría el tránsito de Venus y advirtió a sus amigos de lo que iba a pasar. Él vio en este evento una oportunidad para medir el diámetro aparente de Venus. Horrocks utilizó un telescopio para proyectar la imagen de Venus pasando en frente del Sol. Debido a que tuvo que atender importantes responsabilidades en su iglesia, solamente pudo hacer sus observaciones a menos de una hora antes de la puesta del Sol, pudiendo sin embargo observar "el más agradable espectáculo, el objeto de su sanguíneo deseo, una mancha de inusual magnitud y de una forma perfectamente circular". Utilizando un telescopio refractor proyectó sobre un pedazo de papel la silueta del planeta Venus moviéndose enfrente del Sol, midiendo cuidadosamente que el diámetro de Venus era de 1.3 minutos de arco, el cual era 10 veces más pequeño de lo que esperaba. Sus observaciones refinaron los conocimientos humanos de la órbita de Venus. Posteriormente, en 1662, Hevelius incluyó en sus famosas publicaciones astronómicas la observación del tránsito de Venus de 1639, visto por Jeremiah Horrocks desde Inglaterra, agregando también información sobre el tránsito de Mercurio observado en Francia.

En 1677, el astrónomo inglés Edmond Halley (1656-1742) observó un tránsito de Mercurio desde la Isla de Santa Helena, en Francia. A partir de este evento, a Halley se le ocurrió la idea de usar los tránsitos de Mercurio o de Venus para medir la distancia de la Tierra al Sol, conocida en astronomía como la "unidad astronómica" 1 u.a., y a partir de ello la escala del Sistema Solar. Halley esperó hasta 1716 para publicar su artículo, y lo hizo en latín para asegurarse que los astrónomos de toda Europa pudieran entender su idea. De acuerdo con las leyes de Kepler del movimiento planetario, los astrónomos de 1700 s conocían las distancias relativas de los planetas al Sol. Si ellos pudieran solamente medir las distancias absolutas entre dos planetas, ellos podrían fácilmente calcular las distancias alrededor del Sistema Solar, incluyendo la unidad astronómica (u. a.). El artículo de Halley demostraba que, si observadores separados por grandes latitudes podían medir con 
precisión el tiempo de duración del tránsito de Venus registrando los tiempos del segundo y tercer contactos, ellos podrían medir ligeramente diferentes situaciones. Estos resultados se podían convertir en las diferentes longitudes de la trayectoria (o cuerdas) de Venus a medida que cruza el disco del Sol. Usando estas discrepancias, los astrónomos podrían usar el sencillo método de la paralaje para triangular la distancia a Venus. Esto a su vez, conllevaría a la unidad astronómica. Y si los astrónomos pudieran medir la unidad astronómica con precisión, se podría determinar la escala del sistema solar, permitiéndoles medir los diámetros de todos los planetas, y las masas de cada planeta con al menos una luna.

\section{Pareja 1761 - 1769}

Los tránsitos de Venus del siglo XVIII ocurrieron en 1761 y en 1769. Para el tránsito de Venus de 1761, estando en juego la medición de la escala del Sistema Solar, varias fueron las expediciones científicas que se organizaron para hacer la medición del tránsito de Venus desde los dos hemisferios de la Tierra. El tránsito de Venus fue totalmente visible en la parte Central de Europa y Asia, y parcialmente visible en África y Australia. Expediciones de astrónomos muy competentes fueron organizadas como proyectos de cooperación científica internacional, ellos se equiparon con telescopios, relojes de péndulos, y otros instrumentos de calidad. Debido a las guerras entre los países, algunas de estas expediciones de los ingleses no pudieron realizarse como se planificaron. El astrónomo Charles Mason y el agrimensor Jeremiah Dixon, después de varias complicaciones pudieron realizar un viaje logrando llegar a la Ciudad del Cabo de África del Sur, y midieron exitosamente la segunda mitad del tránsito de Venus, siendo esta la única observación reportada del hemisferio sur. Los franceses, empleando diferentes métodos de triangulación enviaron cuatro expediciones. El astrónomo Jean Baptiste Chappe dÁuteroche (1722-1769) dirigió una expedición al pueblo ruso de Tobolsk, al este de los Montes Urales. Otra expedición fue dirigida por el astrónomo Guillaume Joseph Hyacinthe Jean-Baptiste Le Gentil de la Galaisiere (1725-1792), quien observó el tránsito de Venus desde el Océano Indico; sin embargo, al observar desde el mar, el movimiento de las olas no le permitió hacer mediciones de precisión científica.

Por otra parte, se atribuye al científico ruso Mikhail Lomonosov (17111765) haber observado el tránsito de Venus desde San Petersburgo, utilizando un telescopio refractor de 4.5 pulgadas. De estas observaciones él reportó haber observado un delgado arco de luz alrededor de Venus durante su ingreso y egreso al disco solar. Este arco se interpretó como la refracción a través de una densa 
atmósfera del planeta, similar o mayor que la de la Tierra.

Fue reconocido Thobern Bergman por el resultado de sus observaciones astronómicas, al haber dado cuenta del efecto de la Gota Negra observada durante el evento del tránsito de Venus. Este es un efecto que ocurre durante el segundo y tercer contactos, cuando Venus es visible sobre la superficie solar tocando el limbo solar. Este efecto de la gota negra impidió desde luego hacer las mediciones precisas para medir la escala del Sistema Solar.

El siguiente tránsito de Venus observado es el de 1769. Para el tránsito de Venus del 3 y 4 de junio de 1769, los astrónomos del mundo nuevamente se entusiasmaron para hacer expediciones en busca del mejor sitio que ofreciera las condiciones más favorables para realizar las observaciones astronómicas. Una de las expediciones más grandes y más famosas fue la que dirigió el Capitán James Cook, acompañado del astrónomo Charles Green y el Botánico Joseph Banks. Ellos viajaron desde Inglaterra hasta Tahití, desde 1768 hasta 1771, en un largo viaje equivalente al de las exploraciones espaciales de nuestros tiempos. Las observaciones se hicieron en el Punto Venus, cerca de Papeete, Tahití en la Polinesia Francesa, este punto actualmente existe y está marcado. La misión de la expedición fue "observar un raro tránsito de Venus a través del Sol, con el propósito de determinar mejor el valor la Unidad Astronómica, esto es la distancia entre la Tierra y el Sol, y a partir de ello la escala absoluta del Sistema Solar". Otras expediciones destacadas realizadas para observar el tránsito de Venus fueron hechas por Pingré a Santo Domingo, por Chappe al Cabo de San Lucas en Baja California y por Le Gentil a Pondicherry en la India. Cook y Green publicaron en 1771 el efecto de la Gota Negra de sus observaciones del tránsito de Venus de 1769. En el diario del Capitán Cook, el 3 de junio de 1769 escribió lo siguiente:

"este día resultó muy favorable para nuestros propósitos como lo podíamos haber deseado, ni una Nube vista durante todo el día y el Aire era perfectamente claro, de manera que tuvimos cada ventaja que nosotros podíamos desear al observar el completo paso del Planeta Venus sobre el disco del Sol: Nosotros vimos muy claramente una Atmósfera o la oscura sombra alrededor del cuerpo del Planeta la cual estaba muy perturbada en los tiempos de los Contactos particularmente los dos internos. El Dr. Solander observó así como el Señor Green y yo mismo, y nosotros diferimos uno del otro en los tiempos de observación de los Contactos mucho más de lo que pudo ser esperado. El telescopio del Señor Green y el mío tenían el mismo poder de magnificación pero el del Doctor era más grande que el nuestro. Fue casi completamente calmo todo el día y el Termómetro expuesto al Sol 
cerca de la mitad del día se elevó un grado de calor que antes no habíamos encontrado."

Los reportes de las expediciones de 1761 y 1769 que observaron el tránsito de Venus dieron a los astrónomos su primer buen valor de la distancia al Sol. Sin embargo, los tiempos precisos no fue posible determinarlos, por el efecto de la "gota negra", un efecto por el cual el borde del disco de Venus aparecía deformarse y ligarse al limbo del Sol.

\section{Pareja 1874 - 1882}

El siguiente par de tránsitos de Venus observados ocurrieron en 1874 y en 1882. El siglo XIX trajo avances tecnológicos significativos respecto a las observaciones previas de los tránsitos de Venus, particularmente en las áreas de construcción de telescopios, fotografía, relojes, y la determinación de la posición global, específicamente de la longitud. Los viajes eran también mucho más rápidos y seguros, y las expediciones podían utilizar el telégrafo para comunicarse rápidamente alrededor del mundo. El tránsito de Venus del 9 de diciembre de 1874 fue visible completamente desde Australia, el lado oeste del Océano Pacífico, Japón, el centro de Asía y la India. Parcialmente fue visto desde Siberia, África excepto la parte oeste, la parte oeste de Asia y el lado este de Europa. Los astrónomos de una docena de países del mundo hicieron grandes esfuerzos y expediciones para medir con mayor precisión el tránsito de Venus. Entre ellos, los astrónomos de Estados Unidos organizados por el Observatorio Naval fueron a Vladivostok, a Nagasaki y a Pekín en el Hemisferio Norte, así como a la Isla de Kerguelen, Hobart y Cambelltown en Tasmania, Queenstown en Nueva Zelandia y las Islas Chatham en el Hemisferio Sur. Los astrónomos británicos fueron al Cairo, la Isla Rodríguez, Christchurch, la Isla Kerguelen, Honolulu, y Mauritius. Muchas de las observaciones fueron hechas a lo largo de Rusia y los observadores franceses fueron a Saigon, Peking, Nagasaki, la Isla de Campbell, la Isla de San Paul y Noumea. El objetivo principal de estas expediciones fue observar, registrar y fotografiar el instante exacto de los contactos internos de Venus con el disco solar. Los resultados de los observadores británicos sugirieron una paralaje solar entre 8.75 y 8.88 segundos de arco. Las observaciones del tránsito de Venus de 1874 desde Australia fueron reportadas por Henry Chamberlain Russel, se publicaron en un libro que también incluyó una imagen de 1761 del efecto de la gota negra de Thobern Bergman en las Transacciones Filosóficas de la Real Sociedad. 
El tránsito de Venus de 1882 ocurrió el 6 de diciembre. Fue documentado en el Periódico de Londres The Sun, que ilustró la noticia presentando algunas humorísticas concepciones artísticas. Este tránsito fue fotografiado en el Vassar College de Estados Unidos por María Mitchell y sus estudiantes. El Observatorio Naval de los Estados Unidos (USNO), realizó expediciones para observar el tránsito de Venus de 1882, documentando el evento con un grande número de placas fotográficas. En la preparación de la Expedición, el USNO imprimió un libro con las "Instrucciones para ver el Tránsito de Venus de 1882". En la publicación se listaba de manera detallada los deberes de cada uno de los miembros de la expedición: Para el momento crítico cuando se observe, trate de medir el tiempo cuando Venus toque el borde interior del Sol, y un extraño fenómeno tal como el efecto de la gota negra repentinamente emerja. Este libro se basó en los resultados de la expedición de ocho años antes de 1874; explicó lo que un observador podía esperar, ver, y como discernir el instante del contacto.

\section{Pareja 2004 - 2012}

Para la ocurrencia de los tránsitos de Venus del 8 de junio de 2004 y el 5 de junio de 2012, cambios científicos y tecnológicos mucho más grandes habían tenido lugar. Muy atrás había quedado el problema de determinar la escala del Sistema Solar, que ahora ya se había resuelto utilizando mediciones de radar y otras técnicas para establecer nuestra distancia al Sol y a otros objetos celestes. La Unión Astronómica Internacional (IAU) en 2012 reconoció que una Unidad Astronómica es la distancia media entre la Tierra y el Sol, siendo igual a 149,597,870.700 kilómetros (o 149,597,870,700 metros). De igual manera, el problema de la gota negra tenía una explicación. A partir de observaciones desde el espacio de un tránsito de Mercurio por el Explorador de la Región de Transición y la Corona Solar (TRACE) de la NASA, parecía que el efecto de la gota negra podía ser atribuido a limitaciones de los telescopios, combinado con el hecho de que el disco solar parece más oscuro en el limbo. También, por diferentes investigaciones y exploraciones espaciales al planeta Venus se comprobó que efectivamente tiene una atmósfera, que ésta es rica en dióxido de carbono, y que ejerce sobre la superficie del planeta una presión de 90 veces la presión atmosférica de la Tierra.

Con modernos telescopios solares, para el tránsito de Venus de 2004 se capturaron imágenes sin precedentes de Venus sobre el fondo del activo Sol. Estos telescopios transmitieron imágenes de Venus transitando la corona solar, atravesando los enormes filamentos magnéticos que en cada momento parecía le devorarían completamente. El planeta se miraba apenas como un muy pequeño punto negro en movimiento. 
Para el tránsito de Venus de junio de 2012, se destacó que el paso de Venus sobre la superficie del Sol produciría una silueta que nadie que hoy está vivo probablemente volvería a ver de nuevo, pero que afortunadamente el evento sería ampliamente visible en los siete continentes, incluida la Antártida. Por la experiencia reciente del Tránsito de 2004 y siendo uno de los eventos más publicitados, se esperaba como efectivamente ocurrió, que millones de personas en diferentes partes del mundo intentarían observarlo por ellas mismas, por lo que también insistentes y precisas advertencias se hicieron sobre las precauciones que debían tomarse en las observaciones solares, aun cuando el cielo estuviese nublado.

El tránsito de Venus fue internacionalmente seguido en tiempo real, mediante trasmisiones en vivo hechas por el Exploratorium de Mauna Kea, Hawaii, Estados Unidos, que presentó observaciones desde el Observatorio de Dinámica Solar de la NASA que desde el 2010 se había puesto en órbita. Las imágenes transmitidas por este Observatorio presentaron al Sol visto a través de un ensamble de cámaras de imágenes atmosféricas a borde del telescopio espacial solar. Las imágenes del tránsito de Venus fueron transmitidas mostrando al Sol con seis tipos de filtros que le daban falsos colores en: 1) Rojo, con longitud de onda de 304 angstroms en ión de Helio, mostrando la cromosfera y la región de transición a una temperatura de 143,000 F; 2) Dorado, con longitud de onda de 171 angstroms en ión de Hierro, mostrando la Corona Quieta y región de transición superior, a una temperatura de 1,800,000 F; 3) Verde azulado, con longitud de onda de 131 angstroms en ión de Hierro, mostrando las regiones de fulguraciones del Sol, a una temperatura de 1,800,000 F; 4) Bronce, con longitud de onda de 193 angstroms en ión de Hierro, mostrando la Corona y fulguraciones de plasma caliente, a una temperatura de 2,700,000 F; 5) Azul: con longitud de onda de 335 angstroms en ión de Hierro, mostrando la región de la Corona Activa, a una temperatura de 9,000,000 F; 6) Violeta, con longitud de onda 211 angstroms en ión de Hierro, mostrando la Región de la Corona Activa, a 3,600,000 F.

La División de Exploración del Sistema Solar de la NASA divulgó boletines explicando que el tránsito o paso de un planeta a través del disco del Sol era una clase especial de eclipse. Vistos desde la Tierra, los tránsitos de los planetas interiores de Mercurio y Venus son posibles. Son eventos más raros que los eclipses del Sol por la Luna que ocurren varias veces en el año, dado que en promedio ocurren solo unos 13 tránsitos de Mercurio en un siglo, necesitándose más de un siglo para que ocurra una pareja de tránsitos de Venus. La Agencia Espacial Europea (ESA) también divulgó explicaciones sobre del tránsito de Venus. Destacó que el tránsito de Venus de 2012 era el primero de la historia en el que hubo un satélite en órbita al planeta, la sonda Venus Express de la ESA. 
El tránsito de Venus de 2012 ofreció a los científicos la oportunidad de observar la disminución de la energía solar durante el paso del planeta. Desde ésta última década, los tránsitos son usados para detectar la existencia de exoplanetas o planetas más allá del Sistema Solar. El telescopio espacial Kepler de la NASA está midiendo el brillo de las estrellas distantes cuando un planeta pasa o transita enfrente de ella. A partir de los datos del tránsito, los científicos pueden determinar el tamaño de los planetas, la longitud de su año y calcular la distancia del planeta desde su estrella. La misión Kepler ha confirmado 61 planetas y más de 2,300 candidatos a planetas usando el método del tránsito. El objetivo científico de la Misión Kepler es medir la estructura y diversidad de los sistemas planetarios de otras estrellas. Una vez detectado, el tamaño de la órbita del planeta puede ser calculado a partir del período (el tiempo que le toma al planeta orbitar alrededor de la estrella) y la masa de la estrella usando la Tercera Ley de Kepler del movimiento planetario. El tamaño del planeta se encuentra de la profundidad del tránsito (cuando decae el brillo de la estrella) y el tamaño de la estrella. A partir del tamaño orbital y la temperatura de la estrella, las características de la temperatura del planeta pueden ser calculadas. A partir de esto, la pregunta de si o no el planeta es habitable (no necesariamente inhabitado) puede ser respondida.

\section{Observaciones del tránsito en Mayapán}

Un tránsito de Venus es un fenómeno por el cual el disco del planeta Venus pasa como una pequeña mancha sobre el Sol. Puede ser visto con el ojo a simple vista (lo cual no es recomendado) notándose como una mancha en movimiento sobre el Sol. A diferencia de las manchas solares que les toma varias semanas moverse sobre el disco Solar, a Venus le toma solamente una seis horas.

Desde los tiempos más remotos, las diferentes civilizaciones observaron con contemplación y detenimiento el cielo. Entre los objetos observados, excepto por el Sol y la Luna, Venus ha sido el más brillante del cielo, por lo que se sabe que desde hace miles de años, los Griegos, Egipcios, Babilonios y los Chinos observaron a Venus y registraron sus movimientos. En Mesoamérica, los Mayas también observaron con mucha precisión los movimientos de Venus, así como sus apariciones y desapariciones sobre el horizonte. Los indicios de sus observaciones están presentes en los glifos hallados en las esculturas y códices. Venus ha sido conocido como dos cuerpos diferentes, la estrella de la tarde, y la estrella de la mañana.

Es posible que un tránsito de Venus pudo haber sido visto justo después de la salida del Sol o justo antes de la puesta del Sol por observadores que no 
tenían acceso a las modernas ayudas de observación como los filtros solares, aluminizados 0 aún vidrios ahumados. Durante el Coloquio de la Unión Astronómica Internacional (IAU) 196: "Tránsito de Venus: Nuevas vistas del Sistema Solar y la Galaxia", el Doctor Jesús Galindo de la Universidad Nacional Autónoma de México sugirió que los mayas pudieron haber visto un tránsito de Venus. Murales y pinturas que se cree se hicieron entre 1200 a 1350 DC supuestamente muestran que "Venus" se consume por el Sol. Si esta premisa se acepta, entonces una parte del tránsito de 1275 pudo haber sido visto por los observadores Mayas desde la ciudad de Mayapán, en México, justo antes de la puesta del Sol del 25 de mayo. Desde este lugar el tránsito de Venus solamente fue observado parcialmente antes de la puesta del Sol.

\section{Retos para Honduras}

La ocurrencia del tránsito de Venus del 5 de junio de 2012, representó para Honduras, y particularmente para la Facultad de Ciencias Espaciales, varios retos, que fueron asumidos con bastantes competencias.

El primer reto tuvo que ver con la correcta información y la debida motivación a la población. El Departamento de Astronomía y Astrofísica, después de haber realizados los cálculos precisos de las circunstancias del observación del tránsito de Venus en Honduras y revisado las condiciones meteorológicas del día del evento, preparó boletines informativos los cuales divulgó en la página web de la Facultad de Ciencias Espaciales, por radio, prensa, televisión y las redes sociales. Un sin número de conferencias fueron dictadas a estudiantes de escuelas y colegios, y al público en general, participando también en una gran cantidad de programas en vivo de radio y televisión.

El segundo reto tuvo que ver con la divulgación de advertencias y medidas seguras de como observar el tránsito de Venus. Los profesores de la Facultad de Ciencias Espaciales, particularmente de los Departamentos de Astronomía y Astrofísica y de Arqueoastronomía y Astronomía Cultural, diseñaron instrumentos sencillos y divulgaron entre estudiantes y público en general como utilizarlos de manera segura para observar el tránsito de Venus. Además, diferentes técnicas y métodos de observación del Sol como las proyecciones y el uso de filtros solares fueron ilustrados, haciendo énfasis en evitar mirar directamente al Sol porque podía causar daños inmediatos e irreparables en los ojos. En la Facultad de Ciencias Espaciales, en las instalaciones del Observatorio Astronómico Centroamericano de Suyapa, el día del tránsito de Venus se recibió casi un millar de personas de todas las edades. Todos los medios de comunicación de la capital se hicieron presentes 
para cubrir la noticia. A lo largo de la tarde, de manera continua los profesores estuvieron impartiendo charlas, y se hicieron enlaces en vivo con el Exploratorium de la NASA para transmitir en tiempo real imágenes del tránsito de Venus obtenidas por el Observatorio de Dinámica Solar. Las condiciones de observación no permitieron hacer observaciones directas desde el Observatorio Astronómico Centroamericano de Suyapa, dado que el cielo se mostró completamente nublado a la hora del tránsito de Venus. Sin embargo, con mucha satisfacción fue posible mostrar a la población las imágenes obtenidas por el equipo de Profesores de la Facultad de Ciencias Espaciales, que se trasladó a la costa norte de Honduras donde el cielo estuvo completamente despejado.

El tercer reto que se tuvo que enfrentar fue, garantizar la obtención de imágenes de los momentos del tránsito de Venus visto desde Honduras. Estas serían las primeras imágenes de la historia de un tránsito de Venus tomadas desde territorio hondureño. Habiendo estudiado con mucho detenimiento las condiciones meteorológicos del país, un equipo de Profesores de los Departamentos de Astronomía y Astrofísica y de Arqueoastronomía y Astronomía Cultural, se trasladó a la costa norte de Honduras, específicamente a Sambo Creek, La Ceiba, Atlántida (Latitud: $15^{\circ} 47^{\prime} 47^{\prime \prime} \mathrm{N}$; Longitud: $86^{\circ} 37^{\prime} 15^{\prime \prime} \mathrm{W}$ ), donde las condiciones de observación eran completamente favorables. Ellos utilizaron cámaras fotográficas montadas sobre telescopios y aparatos GPS, y una vez instalado su puesto de observación obtuvieron una secuencia de imágenes desde el momento en que inició el tránsito de Venus hasta la hora en que se puso el Sol. De esta manera han quedado documentados el I y II Contactos del tránsito de Venus vistos desde Honduras.

En Honduras, el impacto total del evento del Tránsito de Venus fue cuantificado por los medios de comunicación en un $10 \%$ de la población, es decir unas 800,000 personas observaron o estuvieron informadas de la ocurrencia de este raro e importante evento astronómico. Esta es la cifra del número de personas que los observadores de 2125 tendrán que superar.

\section{Bibliografía}

- Anzures Becerrill, E., Hernández Santisteban, J. V., \& Farah Simón, A. (2012). Tránsito de Venus, El último del siglo, 5 dejunio de 2012. México: Sociedad Astronómica de México, Instituto de Astronomía, Universidad Nacional Autónoma de México, Academia Mexicana de Ciencias. 
- ESA. (25 de Mayo de 2012). ESPAÑA. Obtenido de Prepárate para el último Tránsito de Venus del siglo: http://www.esa.int/esl/ESA_in_your_country/ Spain/!Preparate_para_el_ultimo_transito_de_Venus_del_siglo

- Espenak, F. (20 de Mayo de 2012). NASA Eclipse web site. Obtenido de Planetary Transits across the Sun: http://eclipse.gsfc.nasa.gov/transit/transit.html

- Espenak, F. (20 de Mayo de 2012). NASA Eclipse web site - GSFC Solar System Exploration Division. Obtenido de 2014 and 2012 Transits of Venus: http:// eclipse.gsfc.nasa.gov/transit/venus0412.html

- Exploratorium. (20 de Mayo de 2012). The rarest eclipse: Transit of Venus. Obtenido de What is a Transit of Venus: http://www.exploratorium.edu/venus/ question1.html

- Fred Espenak, NASA/GSFC. (20 de Mayo de 2012). NASA Eclipse web site. Obtenido de 2004 and 2012 Transit of Venus: http://eclipse.gsfc.nasa.gov/transit/venus0412.html

- Galindo Trejo, J., \& Allen, C. (2005). Maya observations of 13th century Transit of Venus. (D. W. Kurtz, Ed.) Transit of Venus: New Views of the Solar System and Galaxy, IAU Colloquium (196), 124-137.

- Naeye, R. (20 de Mayo de 2012). Transits of Venus in History. Sky \& Telescope, Part 1, Part 2 , Part 3.

- NASA. (20 de mayo de 2012). Kepler. Obtenido de Kepler Overview: http:// www.nasa.gov/mission_pages/kepler/overview/index.html\#.VMQkTf6G-VA

- $\quad$ NASA. (5 de June de 2012). NASA. Obtenido de Web Chat/Streaming Video: Venus Transit Live on June 5: http://www.nasa.gov/connect/chat/venus_transit. html\#.VMQezf6G-VA

- Office, HM Nautical Almanac. (20 de Mayo de 2012). HM Nautical Almanac Office. Obtenido de HM Nautical Almanac Office: Transit of Venus: http://astro. ukho.gov.uk/nao/transit/ 
- Pasachoff, J. M. (20 de mayo de 2012). The Phy Betta Kappa Sociedad, The nation oldest Academic Honor Society, USA. Obtenido de 2011 Phy Betta Kappa Video Series"The june 5, 2012, Transit of Venus" from Jay M. Pasachoff: http://www.pbk.org/home/playpodcast.aspx?id=772

- Pasachoff, J. M. (20 de mayo de 2012). The transit of Venus and Mercury. Obtenido de Transit of Venus home: http://web.williams.edu/Astronomy/eclipse/ transits/index.htm\#TransitofVenusHome

- Phillips, T. (20 de Mayo de 2012). NASA. Obtenido de The 2012 Transit of Venus, Science@NASA: http://www.nasa.gov/mission_pages/sunearth/ news/2012-venus-transit.html

- Solar Dyamics Observatory. (20 de Mayo de 2012). SDO/ Mission. Obtenido de About The SDO Mission: http://sdo.gsfc.nasa.gov/mission/

- Transit of venus.org. (20 de Mayo de 2012). transit of venus.org. Obtenido de History: Centuries of Discovery: http://www.transitofvenus.org/history

- United States Naval Observatory. (20 de Mayo de 2012). Data Services. Obtenido de Transit Computer: http://aa.usno.navy.mil/data/docs/Transit.php 